TAIWANESE JOURNAL OF MATHEMATICS

Vol. 14, No. 2, pp. 541-559, April 2010

This paper is available online at http://www.tjm.nsysu.edu.tw/

\title{
ITERATIVE ALGORITHMS FOR NONEXPANSIVE MAPPINGS ON HADAMARD MANIFOLDS
}

\author{
Chong $\mathrm{Li}^{1, *}$, Genaro Lopez ${ }^{2}$ and Victoria Martín-Márquez ${ }^{3}$
}

\begin{abstract}
Two iterative algorithms for nonexpansive mappings on Hadamard manifolds, which are extensions of the well-known Halpern's and Mann's algorithms in Euclidean spaces, are proposed and proved to be convergent to a fixed point of the mapping. Some numerical examples are provided.
\end{abstract}

\section{INTRODUCTION}

Many practical problems can be formulated as a fixed point problem

$$
x=T x,
$$

where $T$ is a nonlinear operator. The solutions of this equation are called fixed points of $T$. If $T$ is a self-contraction defined on a complete metric space $X$, Banach contraction principle sets up that, for any $x \in X$, the sequence of iterates $\left\{T^{n} x\right\}$ converges strongly to a solution to (1.1). If the mapping $T$ is nonexpansive we must assume additional conditions to assure the existence of fixed point of $T$ and, even when a fixed point exists, the sequence of iterates in general does not converge to a fixed point. This fact has motivated that the study of the asymptotic behavior of nonexpansive mappings is one of the most active research areas in nonlinear analysis. Most of the research have focused on the case when $T$ is a self-mapping defined on a convex subset $K$ of a normed linear space and basically two types of algorithms has been considered: Halpern's and Mann's algorithms.

Received April 1, 2008, accepted September 30, 2008.

Communicated by J. C. Yao.

2000 Mathematics Subject Classification: 47H09, 47H14, 65K05, 90C25.

Key words and phrases: Hadamard manifold, Nonexpansive mapping, Fixed point, Iterative algorithm.

${ }^{1}$ Supported in part by DGES, Grant SAB 2006-0195, Spain; the National Natural Science Foundations of China (Grant No. 10671175; 10731060).

${ }^{2}$ Supported by DGES, Grant MTM2006-13997-C02-01 and Junta de Andalucía, Grant FQM-127.

${ }^{3}$ Supported by Junta de Andalucía, Grant FQM-127 and Ministerio de Ciencia e Innovación, Grant AP 2005-1018.

*Corresponding author. 
Let $u, x_{0} \in K$ and a sequence $\left\{\alpha_{n}\right\} \subset(0,1)$ be given. Halpern's algorithm presented in [12] generates a sequence via the formula

$$
x_{n+1}=\alpha_{n} u+\left(1-\alpha_{n}\right) T x_{n}, \quad n \geq 0 .
$$

A strong effort has been dedicated to the study of the convergence of this sequence, see $[12,18,30,24,31,3,27]$ and references therein, but the full picture is still not clear (cf.[32]).

Mann's algorithm generates a sequence via the following iteration

$$
x_{n+1}=\alpha_{n} x_{n}+\left(1-\alpha_{n}\right) T x_{n}, \quad n \geq 0 .
$$

In the case when a fixed point exists, while Halpern iteration converges strongly, we just get weak convergence for Mann iteration. See [9] for example. Conditions for the convergence of the algorithm can be found in [19, 13, 23, 8, 31]. Some modifications have been proposed in $[20,14]$ to get strong convergence.

Because of the convex structure of both algorithms, few results have been obtained out of the setting of linear spaces. Our interest here is to develop a theory of fixed point approximation for nonexpansive mappings in Hadamard manifolds (i.e. complete simply connected Riemannian manifolds of nonpositive curvature). Extensions to Riemannian manifolds of concepts and techniques which fit in Euclidean spaces are natural. Actually, in the recent years, some algorithms for solving variational inequalities and minimization problems which involves monotone operators have been extended from the Hilbert space framework to the more general setting of Riemannian manifolds (cf. [28, 5, 21, 6, 7]).

For Mann's algorithm, some results had been obtained previously in Hyperbolic metric spaces, see $[15,10,11,25]$. This class of spaces includes Hadamard manifolds and the Hilbert ball equipped with the hyperbolic metric as special cases. For Halpern's algorithm the only precedent, as far as we know, is a result in [16] by W. A. Kirk of convergence for an implicit algorithm in the more abstract setting of geodesic spaces.

The organization of the paper is as follows. In section 2 we introduce some basic concepts, results and notations on Riemannian manifolds. In sections 3 and 4, we prove some properties of the metric defined on Hadamard manifolds and convergence results for Halpern's and Mann's algorithms. Finally, section 5 is devoted to some numerical examples to illustrate applications of the results in the present paper.

\section{Preliminaries}

In this section we introduce some fundamental definitions, properties and notations of Riemannian manifolds, which can be found in any textbook, for example, $[4,26]$. 
Let $M$ be a connected $m$-dimensional manifold and let $p \in M$. We use $T_{p} M$ to denote the tangent space of $M$ at $p$. We always assume that $M$ can be endowed with a Riemannian metric $\langle.,$.$\rangle , with the corresponding norm denoted by \|$.$\| , to$ become a Riemannian manifold. Given a piecewise smooth curve $c:[a, b] \rightarrow M$ joining $p$ to $q$ (i.e. $c(a)=p$ and $c(b)=q$ ), we define the length of $c$ by using the metric as $L(c)=\int_{a}^{b}\left\|c^{\prime}(t)\right\| d t$. Then the Riemannian distance $d(p, q)$ is defined to be the minimal length over the set of all such curves joining $p$ to $q$, which induces the original topology on $M$.

Let $\nabla$ be the Levi-Civita connection associated to $(M,\langle\rangle$,$) . Let c$ be a smooth curve. A smooth vector field $X$ along $c$ is said to be parallel if $\nabla_{c^{\prime}} X=0$. If $c^{\prime}$ itself is parallel, we say that $c$ is a geodesic, and in this case $\left\|c^{\prime}\right\|$ is constant. Furthermore, if $\left\|c^{\prime}\right\|=1, c$ is called normalized. A geodesic joining $p$ to $q$ in $M$ is said to be a minimal geodesic if its length equals $d(p, q)$.

A Riemannian manifold is complete if for any $p \in M$ all geodesics emanating from $p$ are defined for all $-\infty<t<\infty$. By the Hopf-Rinow Theorem we know that if $M$ is complete then any pair of points in $M$ can be joined by a minimizing geodesic. Moreover, $(M, d)$ is a complete metric space, and bounded closed subsets are compact.

Assuming that $m$ is complete, the exponential map $\exp _{p}: T_{p} M \rightarrow M$ at $p \in M$ is defined by $\exp _{p} v=\gamma_{v}(1, p)$ for each $v \in T_{p} M$, where $\gamma()=.\gamma_{v}(., p)$ is the geodesic starting at $p$ with velocity $v$. Then $\exp _{p} t v=\gamma_{v}(t, p)$ for each real number $t$.

A complete simply connected Riemannian manifold of nonpositive sectional curvature is called a Hadamard manifold. Throughout the remainder of the paper, we will always assume that $M$ is a $m$-dimensional Hadamard manifold. The following result is well-known and will be useful.

Proposition 2.1. [26]. Let $p \in M$. Then $\exp _{p}: T_{p} M \rightarrow M$ is a diffeomorphism, and for any two points $p, q \in M$ there exists a unique normalized geodesic joining $p$ to $q$, which is in fact a minimal geodesic.

This proposition says that $M$ is diffeomorphic to the Euclidean space $\mathbb{R}^{m}$. Then, $M$ has the same topology and differential structure as $\mathbb{R}^{m}$. Moreover, Hadamard manifolds and Euclidean spaces have some similar geometrical properties. Some of them are described in the following propositions.

A geodesic triangle $\Delta\left(p_{1}, p_{2}, p_{3}\right)$ of a Riemannian manifold is a set consisting of three points $p_{1}, p_{2}$ and $p_{3}$, and three minimal geodesics $\gamma_{i}$ joining $p_{i}$ to $p_{i+1}$, with $i=1,2,3(\bmod 3)$.

Proposition 2.2. [26] (Comparison theorem for triangles). Let $\Delta\left(p_{1}, p_{2}, p_{3}\right)$ be a geodesic triangle. Denote, for each $i=1,2,3(\bmod 3)$, by $\gamma_{i}:\left[0, l_{i}\right] \rightarrow M$ the geodesic joining $p_{i}$ to $p_{i+1}$, and set $l_{i}=L\left(\gamma_{i}\right), \alpha_{i}:=\angle\left(\gamma_{i}^{\prime}(0),-\gamma_{i-1}^{\prime}\left(l_{i-1}\right)\right)$. Then 


$$
\begin{gathered}
\alpha_{1}+\alpha_{2}+\alpha_{3} \leq \pi \\
l_{i}^{2}+l_{i+1}^{2}-2 l_{i} l_{i+1} \cos \alpha_{i+1} \leq l_{i-1}^{2} .
\end{gathered}
$$

In terms of the distance and the exponential map, the inequality (2.2) can be rewritten as

$$
d^{2}\left(p_{i}, p_{i+1}\right)+d^{2}\left(p_{i+1}, p_{i+2}\right)-2\left\langle\exp _{p_{i+1}}^{-1} p_{i}, \exp _{p_{i+1}}^{-1} p_{i+2}\right\rangle \leq d^{2}\left(p_{i-1}, p_{i}\right)
$$

since

$$
\left\langle\exp _{p_{i+1}}^{-1} p_{i}, \exp _{p_{i+1}}^{-1} p_{i+2}\right\rangle=d\left(p_{i}, p_{i+1}\right) d\left(p_{i+1}, p_{i+2}\right) \cos \alpha_{i+1} .
$$

A subset $K \subseteq M$ is said to be convex if for any two points $p$ and $q$ in $K$, the geodesic joining $p$ to $q$ is contained in $K$, that is, if $\gamma:[a, b] \rightarrow M$ is a geodesic such that $p=\gamma(a)$ and $q=\gamma(b)$, then $\gamma((1-t) a+t b) \in K$ for all $t \in[0,1]$. From now $K$ will denote a nonempty, closed and convex set in $M$.

A real-valued function $f$ defined on $M$ is said to be convex if for any geodesic $\gamma$ of $M$, the composition function $f \circ \gamma: \mathbb{R} \rightarrow \mathbb{R}$ is convex, that is,

$$
(f \circ \gamma)(t a+(1-t) b) \leq t(f \circ \gamma)(a)+(1-t)(f \circ \gamma)(b)
$$

for any $a, b \in \mathbb{R}$, and $0 \leq t \leq 1$.

Proposition 2.3. [26]. Let $d: M \times M \rightarrow \mathbb{R}$ be the distance function. Then $d$ is a convex function with respect to the product Riemannian metric, i.e. given any pair of geodesics $\gamma_{1}:[0,1] \rightarrow M$ and $\gamma_{2}:[0,1] \rightarrow M$ the following inequality holds for all $t \in[0,1]$ :

$$
d\left(\gamma_{1}(t), \gamma_{2}(t)\right) \leq(1-t) d\left(\gamma_{1}(0), \gamma_{2}(0)\right)+t d\left(\gamma_{1}(1), \gamma_{2}(1)\right) .
$$

In particular, for each $p \in M$, the function $d(\cdot, p): M \rightarrow \mathbb{R}$ is a convex function.

Let $P_{K}$ denote the projection onto $K$ defined by

$$
P_{K}(p)=\left\{p_{0} \in K: d\left(p, p_{0}\right) \leq d(p, q) \text { for all } q \in K\right\}, \quad \forall p \in M .
$$

Proposition 2.4. [29]. For any point $p \in M, P_{K}(p)$ is a singleton and the following inequality holds for all $q \in K$ :

$$
\left\langle\exp _{P_{K}(p)}^{-1} p, \exp _{P_{K}(p)}^{-1} q\right\rangle \leq 0 .
$$

\section{Halpern's Algorithm in Hadamard Manifolds}

Let $K$ be a closed convex subset of $M$ and $T: K \rightarrow K$ a mapping. We say that 
$T$ is nonexpansive if for any two points $x, y \in K$ the following inequality holds:

$$
d(T(x), T(y)) \leq d(x, y) .
$$

In order to solve the important problem of finding a fixed point of $T$, Kirk provided in [16] an implicit algorithm for approximating fixed points of nonexpansive mappings in more general metric spaces called geodesic spaces which contain the class of Hadamard manifolds. Applying the general result by Kirk to Hadamard manifolds, one has the following result. Let $\operatorname{Fix}(T)$ denote the set of all fixed points of $T$.

Theorem 3.1. [16]. Suppose that $K$ is a bounded closed convex subset of $M$ and $T: K \rightarrow K$ is nonexpansive. Let $x \in K$, and for each $t \in[0,1)$ let $x_{t}$ be the unique point such that

$$
x_{t} \in\left[x, T\left(x_{t}\right)\right] \quad \text { and } \quad d\left(x, x_{t}\right)=t d\left(x, T\left(x_{t}\right)\right)
$$

(which exists by Banach contraction principle). Then $\lim _{t \rightarrow 1^{-}} x_{t}=\bar{x}$, the unique nearest point to $x$ in Fix $(T)$.

In an Euclidean space $\mathbb{R}^{n}$, this iteration scheme turns into $x_{t}=(1-t) x+t T\left(x_{t}\right)$, which coincides with the implicit Browder's iteration:

$$
x_{t}=t x+(1-t) T\left(x_{t}\right),
$$

which, as $t \rightarrow 0^{+}$, converges strongly to the fixed point of $T$ which is the nearest to $x$ in Fix $(T)$, even in any uniformly smooth space (except that the limit is a certain retraction different from the projection). See [2] and [24] for more details.

There exists a lot of research about the convergence of the explicit Halpern's iteration (1.2) in a Banach space $X$. Below we present an analogue of this algorithm for approximating fixed point for nonexpansive mappings on Hadamard manifolds, which coincides with the Halpern's one in the particular case of an Euclidean space. Let $x_{0}, u \in M$ and let $\left\{\alpha_{n}\right\} \subset(0,1)$. Consider the iteration scheme

$$
x_{n+1}=\exp _{u}\left(1-\alpha_{n}\right) \exp _{u}^{-1} T\left(x_{n}\right), \quad \forall n \geq 0 ;
$$

or equivalently,

$$
x_{n+1}=\gamma_{n}\left(1-\alpha_{n}\right), \quad \forall n \geq 0,
$$

where $\gamma_{n}:[0,1] \rightarrow M$ is the geodesic joining $u$ to $T\left(x_{n}\right)$ (i.e. $\gamma(0)=u$ and $\left.\gamma(1)=T\left(x_{n}\right)\right)$.

In order to prove the convergence of the sequence generated by the algorithm (3.1) we first need some lemmas. 
Lemma 3.2. [31]. Let $\left\{\beta_{n}\right\}$ and $\left\{b_{n}\right\}$ be the sequences satisfying the following conditions.

(i) $\left\{\beta_{n}\right\} \subset[0,1], \sum_{n} \beta_{n}=\infty$;

(ii) $\lim \sup _{n \rightarrow \infty} b_{n} \leq 0$.

Let $\left\{a_{n}\right\}$ be a sequence of non-negative real numbers such that

$$
a_{n+1} \leq\left(1-\beta_{n}\right) a_{n}+\beta_{n} b_{n}, n \geq 0 .
$$

Then $\lim _{n \rightarrow \infty} a_{n}=0$.

Lemma 3.3. [1, p. 24]. Let $\Delta(p, q, r)$ be a geodesic triangle in $M$ Hadamard manifold. Then, there exists $p^{\prime}, q^{\prime}, r^{\prime} \in \mathbb{R}^{2}$ such that

$$
d(p, q)=\left\|p^{\prime}-q^{\prime}\right\|, \quad d(q, r)=\left\|q^{\prime}-r^{\prime}\right\|, \quad d(r, p)=\left\|r^{\prime}-p^{\prime}\right\|
$$

The triangle $\Delta\left(p^{\prime}, q^{\prime}, r^{\prime}\right)$ is called the comparison triangle of the geodesic triangle $\Delta(p, q, r)$, which is unique up to isometry of $M$. The following lemma can be proved from element geometry. This is also a direct application of the Alexandrov's Lemma in $\mathbb{R}^{2}$ (see [1, p. 25]).

Lemma 3.4. Consider four distinct points $p, q, r, z \in \mathbb{R}^{2}$. Suppose that $p$ and $q$ lie on opposite sides of the line through $r$ and $z$. Consider the triangles $\Delta(r, p, z)$ and $\Delta(r, q, z)$. Let $\beta$ be the angle of $\Delta(r, q, z)$ at the vertex $q$, and let $\theta_{1}$ and $\theta_{2}$ be the angles of $\Delta(r, p, z)$ and $\Delta(r, q, z)$ at the vertex $z$, respectively. Let $q^{\prime}$ be the point such that $d\left(r, q^{\prime}\right)=d(r, q)$ and $d\left(p, q^{\prime}\right)=d(p, z)+d(z, q)$. Let $\beta^{\prime}$ be the angles of $\Delta\left(r, p, q^{\prime}\right)$ at the vertex $q^{\prime}$ (see Figure 3.1). If $\theta_{1}+\theta_{2} \geq \pi$, then

$$
\beta \leq \beta^{\prime}
$$

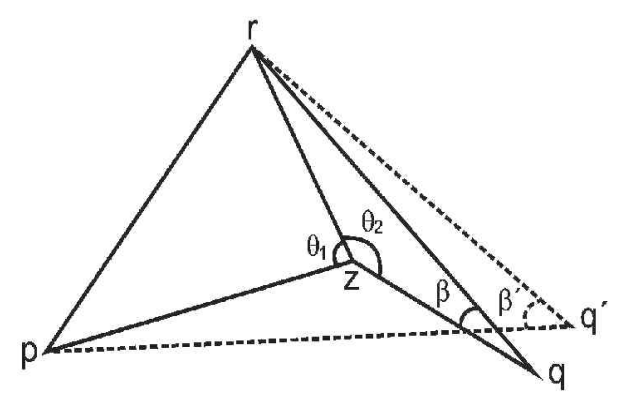

Fig. 3.1 
The next result shows the relation between a geodesic triangle and its comparison triangle involving angles and distances between points.

Lemma 3.5. Let $\Delta(p, q, r)$ be a geodesic triangle in a Hadamard manifold $M$ and $\Delta\left(p^{\prime}, q^{\prime}, r^{\prime}\right)$ be its comparison triangle.

(1) Let $\alpha, \beta, \gamma\left(\right.$ resp. $\left.\alpha^{\prime}, \beta^{\prime}, \gamma^{\prime}\right)$ be the angles of $\Delta(p, q, r)\left(\right.$ resp. $\left.\Delta\left(p^{\prime}, q^{\prime}, r^{\prime}\right)\right)$ at the vertices $p, q, r$ (resp. $\left.p^{\prime}, q^{\prime}, r^{\prime}\right)$. Then, the following inequalities hold:

$$
\alpha^{\prime} \geq \alpha, \quad \beta^{\prime} \geq \beta, \quad \gamma^{\prime} \geq \gamma
$$

(2) Let $z$ be a point in the geodesic joining $p$ to $q$ and $z^{\prime}$ its comparison point in the interval $\left[p^{\prime}, q^{\prime}\right]$. Suppose that $d(z, p)=\left\|z^{\prime}-p^{\prime}\right\|$ and $d(z, q)=\left\|z^{\prime}-q^{\prime}\right\|$, see Figure 3.2. Then the following inequality holds:

$$
d(z, r) \leq\left\|z^{\prime}-r^{\prime}\right\|
$$
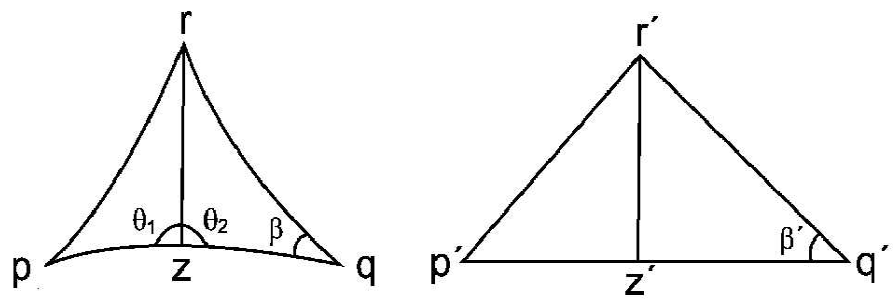

Fig. 3.2

Proof.

(1) We only prove the inequality $\beta^{\prime} \geq \beta$. To do this, consider the triangle $\Delta\left(p^{\prime}, q^{\prime}, r^{\prime}\right)$ in $\mathbb{R}^{2}$. Then, by the law of cosines we have that

$$
\left\|r^{\prime}-q^{\prime}\right\|^{2}+\left\|q^{\prime}-p^{\prime}\right\|^{2}-2\left\|r^{\prime}-q^{\prime}\right\|\left\|q^{\prime}-p^{\prime}\right\| \cos \beta^{\prime}=\left\|r^{\prime}-p^{\prime}\right\|^{2} .
$$

By (2.2), one has that

$$
d^{2}(r, q)+d^{2}(q, p)-2 d(r, q) d(q, p) \cos \beta \leq d^{2}(r, p) .
$$

It follows from Lemma 3.3 that

$$
\cos \beta \leq \cos \beta^{\prime},
$$

and $\beta^{\prime}>\beta$ because $\beta, \beta^{\prime} \in[0, \pi]$.

(2) We fix a geodesic joining $z$ to $r$. Let $\theta_{1}$ and $\theta_{2}$ denote respectively the angles of $\Delta(r, p, z)$ and $\Delta(r, q, z)$ at the vertex $z$. Let $\beta$ and $\beta^{\prime}$ be the angles of $\Delta(r, p, q)$ and $\Delta\left(r^{\prime}, p^{\prime}, q^{\prime}\right)$ at the vertex $q$ and $q^{\prime}$ respectively. See Figure 3.2. 
Consider comparison triangles $\Delta(\bar{r}, \bar{p}, \bar{z})$ and $\Delta(\bar{r}, \bar{q}, \bar{z})$ for the geodesic triangles $\Delta(r, p, z)$ and $\Delta(r, q, z)$ respectively, such that they share the same edge $[\bar{r}, \bar{z}]$, and $\bar{p}, \bar{q}$ lie on opposite sides of the line which passes through $\bar{r}$ and $\bar{z}$. Let $\bar{\theta}_{1}$ and $\bar{\theta}_{2}$ be the angles of $\Delta(\bar{r}, \bar{p}, \bar{z})$ and $\Delta(\bar{r}, \bar{q}, \bar{z})$ at the vertex $\bar{z}$, respectively. Denote $\bar{\beta}$ the angle at the vertex $\bar{q}$. From the inequalities (3.2) we deduce that

$$
\bar{\theta}_{1}+\bar{\theta}_{2} \geq \theta_{1}+\theta_{2}=\pi .
$$

Thus Lemma 3.4 is applicable to getting that $\bar{\beta} \leq \beta^{\prime}$. Therefore, using the law of cosines, we have $d(z, r) \leq\left\|z^{\prime}-r^{\prime}\right\|$.

For the main theorem of this section, we consider the following hypothesis:

(H1) $\lim _{n \rightarrow \infty} \alpha_{n}=0$;

(H2) $\sum_{n \geq 0} \alpha_{n}=\infty$;

(H3) $\sum_{n \geq 0}\left|\alpha_{n+1}-\alpha_{n}\right|<\infty$;

(H4) $\lim _{n \rightarrow \infty}\left(\alpha_{n}-\alpha_{n-1}\right) / \alpha_{n}=0$.

Theorem 3.6. Let $K$ be a closed convex subset of $M$ and $T: K \rightarrow K a$ nonexpansive mapping with $F:=F i x(T) \neq \emptyset$. Let $u, x_{0} \in M$. Suppose that $\left\{\alpha_{n}\right\} \in(0,1)$ satisfies $(H 1)$, (H2) and, (H3) or (H4). Then the sequence $\left\{x_{n}\right\}$ generated by the algorithm (3.1) converges to $P_{F}(u)$.

Proof. Let $n \geq 0$ and let $\gamma_{n}:[0,1] \rightarrow M$ denote the geodesic joining $u$ to $T\left(x_{n}\right)$. We divide the proof into four steps.

Step 1. $\left\{x_{n}\right\}$ and $\left\{T\left(x_{n}\right)\right\}$ are bounded.

Since the boundedness of $\left\{T\left(x_{n}\right)\right\}$ is a direct consequence of that of $\left\{x_{n}\right\}$, below we only prove the boundedness of $\left\{x_{n}\right\}$. For this purpose, take $x \in F$ and fix $n$. Then, by the convexity of the distance function and the nonexpansivity of $T$, we have that

$$
\begin{aligned}
d\left(x_{n+1}, x\right) & =d\left(\gamma_{n}\left(1-\alpha_{n}\right), x\right) \\
& \leq \alpha_{n} d\left(\gamma_{n}(0), x\right)+\left(1-\alpha_{n}\right) d\left(\gamma_{n}(1), x\right) \\
& =\alpha_{n} d(u, x)+\left(1-\alpha_{n}\right) d\left(T\left(x_{n}\right), x\right) \\
& \leq \alpha_{n} d(u, x)+\left(1-\alpha_{n}\right) d\left(x_{n}, x\right) .
\end{aligned}
$$

Then the boundness of $\left\{x_{n}\right\}$ is seen to hold by mathematical induction.

Step 2. $\lim _{n \rightarrow \infty} d\left(x_{n+1}, x_{n}\right)=0$.

By Step 1, we can find a constant $C$ such that

$$
d\left(x_{n}, x_{n-1}\right) \leq C \quad \text { and } \quad d\left(u, T\left(x_{n}\right)\right) \leq C, \quad \forall n \geq 0 .
$$


Using the convexity of the distance function, we have that, for each $n \geq 0$,

$$
\begin{aligned}
d\left(x_{n+1}, x_{n}\right)= & d\left(\gamma_{n}\left(1-\alpha_{n}\right), \gamma_{n-1}\left(1-\alpha_{n-1}\right)\right) \\
\leq & d\left(\gamma_{n}\left(1-\alpha_{n}\right), \gamma_{n-1}\left(1-\alpha_{n}\right)\right) \\
& +d\left(\gamma_{n-1}\left(1-\alpha_{n}\right), \gamma_{n-1}\left(1-\alpha_{n-1}\right)\right) \\
\leq & \left(1-\alpha_{n}\right) d\left(T\left(x_{n}\right), T\left(x_{n-1}\right)\right)+\left|\alpha_{n}-\alpha_{n-1}\right| d\left(u, T\left(x_{n-1}\right)\right) .
\end{aligned}
$$

This together with (3.4) and the nonexpansivity of $T$ implies that

$$
d\left(x_{n+1}, x_{n}\right) \leq\left(1-\alpha_{n}\right) d\left(x_{n}, x_{n-1}\right)+C\left|\alpha_{n}-\alpha_{n-1}\right|, \quad \forall n \geq 0 .
$$

Thus, if (H4) holds, we apply Lemma 3.2 (with $\beta_{n}=\alpha_{n}$ and $b_{n}=C\left|\alpha_{n}-\alpha_{n-1}\right| / \alpha_{n}$ for each $n$ ) to conclude that $\lim _{n \rightarrow \infty} d\left(x_{n+1}, x_{n}\right)=0$. As to the case when (H3) holds, let $k \leq n$. By (3.6), one gets that

$$
\begin{aligned}
d\left(x_{n+1}, x_{n}\right) & \leq \prod_{i=k}^{n}\left(1-\alpha_{i}\right) d\left(x_{k}, x_{k-1}\right)+C \sum_{i=k}^{n}\left|\alpha_{i}-\alpha_{i-1}\right| \\
& \leq C \prod_{i=k}^{n}\left(1-\alpha_{i}\right)+C \sum_{i=k}^{n}\left|\alpha_{i}-\alpha_{i-1}\right| .
\end{aligned}
$$

Letting $n \rightarrow \infty$ implies that,

$$
\lim _{n \rightarrow \infty} d\left(x_{n+1}, x_{n}\right) \leq C \prod_{i=k}^{\infty}\left(1-\alpha_{i}\right)+C \sum_{i=k}^{\infty}\left|\alpha_{i}-\alpha_{i-1}\right| .
$$

Condition (H2) implies that $\lim _{k \rightarrow \infty} \prod_{i=k}^{\infty}\left(1-\alpha_{i}\right)=0$; while condition (H3) implies that $\lim _{k \rightarrow \infty} \sum_{i=k}^{\infty}\left|\alpha_{i}-\alpha_{i-1}\right|=0$. Hence, letting $k \rightarrow \infty$ in (3.7), we get $\lim _{n \rightarrow \infty} d\left(x_{n+1}, x_{n}\right)=0$ in the case when (H3) holds.

Step 3. $\lim \sup _{n \rightarrow \infty}\left\langle\exp _{P_{F}(u)}^{-1} u, \exp _{P_{F}(u)}^{-1} T\left(x_{n}\right)\right\rangle \leq 0$.

By Step 1, $\left\{\left\langle\exp _{P_{F}(u)}^{-1} u, \exp _{P_{F}(u)}^{-1} T\left(x_{n}\right)\right\rangle\right\}$ is bounded; hence its upper limit exists. Thus we can find a subsequence $\left\{n_{k}\right\}$ of $\{n\}$ such that

$$
\limsup _{n \rightarrow \infty}\left\langle\exp _{P_{F}(u)}^{-1} u, \exp _{P_{F}(u)}^{-1} T\left(x_{n}\right)\right\rangle=\lim _{k \rightarrow \infty}\left\langle\exp _{P_{F}(u)}^{-1} u, \exp _{P_{F}(u)}^{-1} T\left(x_{n_{k}}\right)\right\rangle .
$$

Without loss of generality, we may assume that $x_{n_{k}} \rightarrow \bar{x}$ for some $\bar{x} \in M$ because $\left\{x_{n}\right\}$ is bounded by Step 1. By the convexity of the distance function and the definition of the algorithm,

$$
d\left(x_{n_{k}+1}, T\left(x_{n_{k}}\right)\right) \leq \alpha_{n_{k}} d\left(u, T\left(x_{n_{k}}\right)\right) .
$$


Since $\left\{d\left(u, T\left(x_{n_{k}}\right)\right)\right\}$ is bounded by Step 1, it follows that $\lim _{k \rightarrow \infty} d\left(x_{n_{k}+1}, T\left(x_{n_{k}}\right)\right)=0$ as $\alpha_{n_{k}} \rightarrow 0$ by (H1). Noting that

$$
d\left(x_{n_{k}}, T\left(x_{n_{k}}\right)\right) \leq d\left(x_{n_{k}+1}, x_{n_{k}}\right)+d\left(x_{n_{k}+1}, T\left(x_{n_{k}}\right)\right),
$$

one sees that $\lim _{n \rightarrow \infty} d\left(x_{n_{k}}, T\left(x_{n_{k}}\right)\right)=0$. Therefore

$$
d(\bar{x}, T(\bar{x})) \leq d\left(\bar{x}, x_{n_{k}}\right)+d\left(x_{n_{k}}, T\left(x_{n_{k}}\right)\right)+d\left(T\left(x_{n_{k}}\right), T(\bar{x})\right) \rightarrow 0,
$$

which means that $\bar{x} \in F$. Then, Proposition 2.4 implies that $\left\langle\exp _{P_{F}(u)}^{-1} u, \exp _{P_{F}(u)}^{-1} \bar{x}\right\rangle \leq 0$ and so

$$
\lim _{k \rightarrow \infty}\left\langle\exp _{P_{F}(u)}^{-1} u, \exp _{P_{F}(u)}^{-1} T\left(x_{n_{k}}\right)\right\rangle=\left\langle\exp _{P_{F}(u)}^{-1} u, \exp _{P_{F}(u)}^{-1} \bar{x}\right\rangle \leq 0 .
$$

Combining this with (3.8), we complete the proof of Step 3.

Step 4. $\lim _{n \rightarrow \infty} d\left(x_{n}, P_{F} u\right)=0$.

Write

$$
b_{n}=\alpha_{n} d\left(u, P_{F} u\right)+\left\langle\exp _{P_{F}(u)}^{-1} u, \exp _{P_{F}(u)}^{-1} T\left(x_{n}\right)\right\rangle, \quad \forall n \geq 0 .
$$

Then $\lim _{n \rightarrow \infty} b_{n} \leq 0$ by Step 3. Thus, by Lemma 3.2, it suffices to verify that

$$
d^{2}\left(x_{n+1}, P_{F} u\right) \leq\left(1-\alpha_{n}\right) d^{2}\left(x_{n}, P_{F} u\right)+\alpha_{n} b_{n}, \quad \forall n \geq 0 .
$$

To this end, we fix $n \geq 0$ and set $p=T\left(x_{n}\right), q=P_{F} u$. Consider the geodesic triangle $\Delta(u, p, q)$ and its comparison triangle $\Delta\left(u^{\prime}, p^{\prime}, q^{\prime}\right)$. Then

$$
d\left(u, P_{F} u\right)=d(u, q)=\left\|u^{\prime}-q^{\prime}\right\| \quad \text { and } \quad d\left(T\left(x_{n}\right), P_{F} u\right)=d(p, q)=\left\|p^{\prime}-q^{\prime}\right\| .
$$

Recall from (3.1) that $x_{n+1}=\exp _{u}\left(1-\alpha_{n}\right) \exp _{u}^{-1} T\left(x_{n}\right)=\exp _{u}\left(1-\alpha_{n}\right) \exp _{u}^{-1} p$. The comparison point of $x_{n+1}$ is $x_{n+1}^{\prime}=\alpha_{n} u^{\prime}+\left(1-\alpha_{n}\right) p^{\prime}$. Let $\beta$ and $\beta^{\prime}$ denote the angles at $q$ and $q^{\prime}$, respectively. Then $\beta \leq \beta^{\prime}$ by Lemma 3.5 (1) and so $\cos \beta^{\prime} \leq \cos \beta$. Then, by Lemma 3.5 (2) we have

$$
\begin{aligned}
& d^{2}\left(x_{n+1}, P_{F} u\right) \\
\leq & \left\|x_{n+1}^{\prime}-q^{\prime}\right\|^{2} \\
= & \left\|\alpha_{n}\left(u^{\prime}-q^{\prime}\right)+\left(1-\alpha_{n}\right)\left(p^{\prime}-q^{\prime}\right)\right\|^{2} \\
= & \alpha_{n}^{2}\left\|u^{\prime}-q^{\prime}\right\|^{2}+\left(1-\alpha_{n}\right)^{2}\left\|p^{\prime}-q^{\prime}\right\|^{2}+2 \alpha_{n}\left(1-\alpha_{n}\right)\left\|u^{\prime}-q^{\prime}\right\|\left\|p^{\prime}-q^{\prime}\right\| \cos \beta^{\prime} \\
\leq & \alpha_{n}^{2} d^{2}\left(u, P_{F} u\right)+\left(1-\alpha_{n}\right)^{2} d^{2}\left(T\left(x_{n}\right), P_{F} u\right) \\
& +2 \alpha_{n}\left(1-\alpha_{n}\right) d\left(u, P_{F} u\right) d\left(T\left(x_{n}\right), P_{F} u\right) \cos \beta \\
\leq & \left(1-\alpha_{n}\right) d^{2}\left(x_{n}, P_{F} u\right)+\alpha_{n}\left(\alpha_{n} d\left(u, P_{F} u\right)+\left\langle\exp _{P_{F}(u)}^{-1} u, \exp _{P_{F}(u)}^{-1} T\left(x_{n}\right)\right\rangle\right) .
\end{aligned}
$$

Hence (3.9) is proved and the proof is complete. 


\section{Mann's Algorithm in Hadamard Manifolds}

In the case when $K$ is a closed convex subset of a normed linear space $X$ and $T: K \rightarrow K$ a nonexpansive mapping, for $\left\{\alpha_{n}\right\} \subset[0,1]$, the iteration process $\left\{x_{n}\right\}$ defined by the algorithm

$$
x_{n+1}=\alpha_{n} x_{n}+\left(1-\alpha_{n}\right) T\left(x_{n}\right), \quad \forall n \geq 0
$$

was introduced by Mann in [19] and is known as Mann's iteration for finding fixed points of nonexpansive mappings. Ishikawa proved in [13] that if $0<a \leq \alpha_{n}<1$ and $\sum_{n=1}^{\infty} \alpha_{n}=\infty$, then $\left\|x_{n}-T\left(x_{n}\right)\right\| \rightarrow 0$ as $n \rightarrow \infty$, which implies the convergence of $\left\{x_{n}\right\}$ to a fixed point of $T$ if the range of $T$ lies in a compact subset of $X$. In 1979, Reich obtained in [23] the weak convergence of the sequence in a uniformly convex space with a Fréchet differentiable norm under the assumption that $\left\{\alpha_{n}\right\} \subset(0,1)$ and $\sum_{n=1}^{\infty} \alpha_{n}\left(1-\alpha_{n}\right)=\infty$. For further results see [8] and references therein.

An extension of Mann's iteration (4.1) and its convergence results to the framework of metric spaces $(X, d)$ is due to Goebel and Kirk [15, 10] and Reich-Shafrir [25], who provided an iterative method for finding fixed points of nonexpansive mappings on spaces of hyperbolic type, which include Hadamard manifolds as special cases. The iteration algorithm is defined by

$$
x_{n+1} \in\left[x_{n}, T\left(x_{n}\right)\right] \text { such that } d\left(x_{n}, T\left(x_{n}\right)\right)=\left(1-\alpha_{n}\right) d\left(x_{n}, x_{n+1}\right),
$$

where $\left\{\alpha_{n}\right\} \subset(0,1)$. In particular, under the assumption that $\left\{\alpha_{n}\right\}$ is bounded away from 0 and 1, Reich and Shafrir proved the convergence of this iteration to a fixed point of $T$. Goebel and Reich [11] studied the behavior of the sequence of the iterates $x_{n+1}=T\left(x_{n}\right)$ in Hyperbolic metric spaces for firmly nonexpansive mappings.

Motivated by these results, we continue to consider Mann's iteration (4.2) in Hadamard manifolds $M$, which has the following form

$$
x_{n+1}=\exp _{x_{n}}\left(1-\alpha_{n}\right) \exp _{x_{n}}^{-1} T\left(x_{n}\right), \quad \forall n \geq 0,
$$

and study the convergence of Mann's iteration (4.2) for nonexpansive mappings $T: M \rightarrow M$ but without assuming that $\left\{\alpha_{n}\right\}$ is bounded away from 0 and 1 . In fact, we will prove in Theorem 4.3 that the sequence $\left\{x_{n}\right\}$ generated by Mann's iteration (4.2) in Hadamard manifolds converges to a fixed point of $T$ only if $\left\{\alpha_{n}\right\}$ satisfies the following condition:

$$
\sum_{n=0}^{\infty} \alpha_{n}\left(1-\alpha_{n}\right)=\infty .
$$

For this purpose, some definitions and results are necessary at first. 
Definition 4.1. Let $X$ be a complete metric space and $F \subset X$ be a nonempty set. A sequence $\left\{x_{n}\right\} \subset X$ is called Fejer convergent to $F$ if

$$
d\left(x_{n+1}, y\right) \leq d\left(x_{n}, y\right)
$$

for all $y \in F$ and $n \geq 0$.

Lemma 4.2. [7]. Let $X$ be a complete metric space. If $\left\{x_{n}\right\} \subset X$ is Fejér convergent to a nonempty set $F \subset X$, then $\left\{x_{n}\right\}$ is bounded. Moreover, if a cluster point $x$ of $\left\{x_{n}\right\}$ belongs to $F$, then $\left\{x_{n}\right\}$ converges to $x$.

Now we are ready to prove the main theorem of this section.

Theorem 4.3. Let $K$ be a closed convex subset of $M$ and $T: K \rightarrow K a$ nonexpansive mapping with $F:=F i x(T) \neq \emptyset$. Suppose that $\left\{\alpha_{n}\right\} \subset(0,1)$ satisfy the condition (4.4). Let $x_{0} \in M$ and let $\left\{x_{n}\right\}$ be the sequence generated by the algorithm (4.3). Then $\left\{x_{n}\right\}$ converges to a fixed point of $T$.

Proof. Note that $K$ itself is a complete metric space. Thus, by Lemma 4.2, it suffices to verify that $\left\{x_{n}\right\}$ is Fejer convergent to $F$ and that all cluster points of $\left\{x_{n}\right\}$ belong to $F$. For this purpose, let $n \geq 0$ and $p \in F$ be fixed. Let $\gamma:[0,1] \rightarrow M$ denote the geodesic joining $x_{n}$ to $T\left(x_{n}\right)$. Then $x_{n+1}=\gamma\left(1-\alpha_{n}\right)$. By the convexity of the distance function and the nonexpansivity of $T$, we have

$$
d\left(x_{n+1}, p\right)=d\left(\gamma\left(1-\alpha_{n}\right), p\right) \leq \alpha_{n} d\left(x_{n}, p\right)+\left(1-\alpha_{n}\right) d\left(T\left(x_{n}\right), p\right) \leq d\left(x_{n}, p\right) .
$$

Hence $\left\{x_{n}\right\}$ is Fejer convergent to $F$. Now let $x$ be a cluster point of $\left\{x_{n}\right\}$. Then there exists a subsequence $\left\{n_{k}\right\}$ of $\{n\}$ such that $x_{n_{k}} \rightarrow x$. Below we prove that

$$
\lim _{n \rightarrow \infty} d\left(x_{n}, T\left(x_{n}\right)\right)=0 .
$$

Granting this, one has that

$$
\begin{aligned}
d(x, T(x)) & \leq d\left(x, x_{n_{k}}\right)+d\left(x_{n_{k}}, T\left(x_{n_{k}}\right)\right)+d\left(T\left(x_{n_{k}}\right), T(x)\right) \\
& \leq 2 d\left(x_{n_{k}}, x\right)+d\left(x_{n_{k}}, T\left(x_{n_{k}}\right)\right),
\end{aligned}
$$

Taking limit, we obtain that $d(x, T x)=0$, which means that $x \in F$, and completes the proof.

To show (4.5), let $p \in F$ and $n \geq 0$. Let $\Delta\left(x_{n}, q, p\right)$ be the geodesic triangle with vertices $x_{n}, q:=T\left(x_{n}\right)$ and $p$. From Lemma 3.3 there exists a comparison triangle $\Delta\left(x_{n}^{\prime}, q^{\prime}, p^{\prime}\right)$ which conserve the length of the edge. Recall 
that $x_{n+1}=\gamma\left(1-\alpha_{n}\right)$. Set $x_{n+1}^{\prime}:=\left(1-\alpha_{n}\right) x_{n}^{\prime}+\alpha_{n} T\left(x_{n}\right)^{\prime}=\left(1-\alpha_{n}\right) x_{n}^{\prime}+\alpha_{n} q^{\prime}$ be its comparison point. By Lemma 3.5 (2),

$$
\begin{aligned}
d^{2}\left(x_{n+1}, p\right) & \leq\left\|x_{n+1}^{\prime}-p^{\prime}\right\|^{2} \\
& =\left\|\alpha_{n}\left(x_{n}^{\prime}-p^{\prime}\right)+\left(1-\alpha_{n}\right)\left(q^{\prime}-p^{\prime}\right)\right\|^{2} \\
& =\alpha_{n}\left\|x_{n}^{\prime}-p^{\prime}\right\|^{2}+\left(1-\alpha_{n}\right)\left\|q^{\prime}-p^{\prime}\right\|^{2}-\alpha_{n}\left(1-\alpha_{n}\right)\left\|x_{n}^{\prime}-q^{\prime}\right\|^{2} \\
& =\alpha_{n} d^{2}\left(x_{n}, p\right)+\left(1-\alpha_{n}\right) d^{2}\left(T\left(x_{n}\right), p\right)-\alpha_{n}\left(1-\alpha_{n}\right) d^{2}\left(x_{n}, T\left(x_{n}\right)\right) \\
& \leq d^{2}\left(x_{n}, p\right)-\alpha_{n}\left(1-\alpha_{n}\right) d^{2}\left(x_{n}, T\left(x_{n}\right)\right) .
\end{aligned}
$$

It follows that

$$
\alpha_{n}\left(1-\alpha_{n}\right) d^{2}\left(x_{n}, T\left(x_{n}\right)\right) \leq d^{2}\left(x_{n}, p\right)-d^{2}\left(x_{n+1}, p\right)
$$

and

$$
\sum_{n=1}^{\infty} \alpha_{n}\left(1-\alpha_{n}\right) d^{2}\left(x_{n}, T\left(x_{n}\right)\right)<\infty
$$

which implies that

$$
\liminf _{n \rightarrow \infty} d\left(x_{n}, T\left(x_{n}\right)\right)=0
$$

because otherwise, $d\left(x_{n}, T\left(x_{n}\right)\right) \geq a \forall n \geq 0$ for some $a>0$, and then,

$$
\sum_{n=1}^{\infty} \alpha_{n}\left(1-\alpha_{n}\right) d\left(x_{n}, T\left(x_{n}\right)\right) \geq a \sum_{n=1}^{\infty} \alpha_{n}\left(1-\alpha_{n}\right)=\infty
$$

which is a contradiction with (4.6).

On the other hand, using the nonexpansivity of $T$ and the convexity of the distance function,

$$
\begin{aligned}
d\left(x_{n+1}, T\left(x_{n+1}\right)\right) & \leq d\left(x_{n+1}, T\left(x_{n}\right)\right)+d\left(T\left(x_{n}\right), T\left(x_{n+1}\right)\right) \\
& \leq d\left(x_{n+1}, T\left(x_{n}\right)\right)+d\left(x_{n}, x_{n+1}\right) \\
& \leq \alpha_{n} d\left(x_{n}, T\left(x_{n}\right)\right)+\left(1-\alpha_{n}\right) d\left(x_{n}, T\left(x_{n}\right)\right) \\
& =d\left(x_{n}, T\left(x_{n}\right)\right) .
\end{aligned}
$$

This means that $\left\{d\left(x_{n}, T\left(x_{n}\right)\right)\right\}$ is a monotone sequence. Combining this and (4.7) completes the proof of (4.5). 


\section{NumERICAL EXAMPLE}

Let $\mathbb{E}^{m, 1}$ denote the vector space $\mathbb{R}^{m+1}$ endowed with the symmetric bilinear form (which is called the Lorentz metric) defined by

$$
\langle x, y\rangle=\sum_{i=1}^{m} x_{i} y_{i}-x_{m+1} y_{m+1}, \quad \forall x=\left(x_{i}\right), y=\left(y_{i}\right) \in \mathbb{R}^{m+1} .
$$

The hyperbolic m-space $\mathbb{H}^{m}$ is defined by

$$
\left\{x=\left(x_{1}, \ldots, x_{m+1}\right) \in \mathbb{E}^{m, 1}:\langle x, x\rangle=-1, x_{m+1}>0\right\},
$$

that is the upper sheet of the hyperboloid $\left\{x \in \mathbb{E}^{m, 1}:\langle x, x\rangle=-1\right\}$. Note that $x_{m+1} \geq 1$ for any $x \in \mathbb{H}^{m}$, with equality if and only if $x_{i}=0$ for all $i=1, \ldots, m$. The metric of $\mathbb{H}^{m}$ is induced from the Lorentz metric $\langle\cdot, \cdot\rangle$ and it will be denoted by the same symbol. Then $\mathbb{H}^{m}$ is a Hadamard manifold with sectional curvature -1 (cf. [1] and [6]). Furthermore, the normalized geodesic $\gamma: \mathbb{R} \rightarrow \mathbb{H}^{m}$ starting from $x \in \mathbb{H}^{m}$ is given by

$$
\gamma(t)=(\cosh t) x+(\sinh t) v, \quad \forall t \in \mathbb{R}
$$

where $v \in T_{x} \mathbb{H}^{m}$ is a unit vector; while the distance $d$ on $\mathbb{H}^{m}$ is

$$
d(x, y)=\operatorname{arccosh}(-\langle x, y\rangle), \quad \forall x, y \in \mathbb{H}^{m} .
$$

Then, the exponential map can be expressed as

$$
\exp _{x}(r v)=(\cosh r) x+(\sinh r) v,
$$

for any $r \in \mathbb{R}^{+}, x \in \mathbb{H}^{m}$ and any unit vector $v \in T_{x} \mathbb{H}^{m}$. To get the expression of the inverse exponential map, we write for any $x, y \in \mathbb{H}^{m}$,

$$
y=\exp _{x}\left(d(x, y) \frac{\exp _{x}^{-1} y}{d(x, y)}\right)=(\cosh d(x, y)) x+(\sinh d(x, y)) \frac{\exp _{x}^{-1} y}{d(x, y)} .
$$

Therefore, by definition of the distance (5.2), we obtain

$$
\exp _{x}^{-1} y=\operatorname{arccosh}(-\langle x, y\rangle) \frac{y+\langle x, y\rangle x}{\sqrt{\langle x, y\rangle^{2}-1}}, \quad \forall x, y \in \mathbb{H}^{m} .
$$

Thus, using these expressions, both algorithms in previous sections can be formulated in a simple way in the hyperbolic space $\mathbb{H}^{m}$. Write

$$
r(x, y)=\operatorname{arccosh}(-\langle x, T(y)\rangle)
$$


and

$$
V(x, y)=\frac{T(y)+\langle x, T(y)\rangle x}{\sqrt{\langle x, T(y)\rangle^{2}-1}} .
$$

Then Halpern's algorithm (3.1) has the form

$$
x_{n+1}=\left(\cosh \left(1-\alpha_{n}\right) r\left(u, x_{n}\right)\right) u+\left(\sinh \left(1-\alpha_{n}\right) r\right) V\left(u, x_{n}\right), \quad \forall n \geq 0 ;
$$

while Mann's algorithm (4.3) has the form

$$
x_{n+1}=\left(\cosh \left(1-\alpha_{n}\right) r\left(x_{n}, x_{n}\right) x_{n}+\left(\sinh \left(1-\alpha_{n}\right) r\right) V\left(x_{n}, x_{n}\right), \quad \forall n \geq 0 .\right.
$$

We end this paper with an example of $\mathbb{H}^{3}$, where these methods are implemented

\begin{tabular}{|c|c|c|c|c|}
\hline \multicolumn{2}{|l|}{$T_{1}$} & $x_{0}^{1}$ & $x_{0}^{2}$ & $x_{0}^{3}$ \\
\hline \multirow{17}{*}{ MANN } & Step & \multicolumn{3}{|c|}{ Error } \\
\hline & $d_{0}$ & 1.23849936794859 & 1.43016443780138 & 0.96560216993144 \\
\hline & $d_{1}$ & 0.61924968397430 & 0.71508221890069 & 0.48280108496572 \\
\hline & $d_{2}$ & 0.37154981038458 & 0.42904933134041 & 0.28968065097943 \\
\hline & $d_{3}$ & 0.24769987358972 & 0.28603288756028 & 0.19312043398629 \\
\hline & $d_{4}$ & 0.17692848113551 & 0.20430920540020 & 0.13794316713307 \\
\hline & $d_{5}$ & 0.13269636085164 & 0.15323190405015 & 0.10345737534980 \\
\hline & $d_{6}$ & 0.10320828066239 & 0.11918036981678 & 0.08046684749429 \\
\hline & $d_{7}$ & 0.08256662452991 & 0.09534429585343 & 0.06437347799544 \\
\hline & $d_{8}$ & 0.06755451097902 & 0.07800896933462 & 0.05266920926899 \\
\hline & $d_{9}$ & 0.05629542581585 & 0.06500747444552 & 0.04389100772417 \\
\hline & $d_{10}$ & 0.04763459107495 & 0.05500632453083 & 0.03713854499737 \\
\hline & Error & \multicolumn{3}{|c|}{ Step } \\
\hline & $10^{-2}$ & $d_{17}$ & $d_{19}$ & $d_{15}$ \\
\hline & $10^{-3}$ & $d_{59}$ & $d_{64}$ & $d_{52}$ \\
\hline & $10^{-4}$ & $d_{191}$ & $d_{205}$ & $d_{168}$ \\
\hline & $10^{-5}$ & $d_{608}$ & $d_{654}$ & $d_{537}$ \\
\hline \multirow{17}{*}{ HALPERN } & Step & \multicolumn{3}{|c|}{ Error } \\
\hline & $d_{0}$ & 1.23849936794859 & 1.43016443780138 & 0.96560216993144 \\
\hline & $d_{1}$ & 0.79109087958575 & 0.94324711126741 & 0.56347787068548 \\
\hline & $d_{2}$ & 0.72937999179719 & 0.83691943183081 & 0.57904469220055 \\
\hline & $d_{3}$ & 0.51644150342379 & 0.61225961625570 & 0.37537998889839 \\
\hline & $d_{4}$ & 0.51460518499467 & 0.58768625280562 & 0.41346210869283 \\
\hline & $d_{5}$ & 0.38173470848514 & 0.45056824179018 & 0.28140064643068 \\
\hline & $d_{6}$ & 0.39667994376475 & 0.45136530320607 & 0.32150418565275 \\
\hline & $d_{7}$ & 0.30206081644163 & 0.35527907593590 & 0.22504211840976 \\
\hline & $d_{8}$ & 0.32231407126604 & 0.36567764790811 & 0.26300078945845 \\
\hline & $d_{9}$ & 0.24953770871435 & 0.29265581960747 & 0.18748437135137 \\
\hline & $d_{10}$ & 0.27120338594617 & 0.30694720797307 & 0.22250671712339 \\
\hline & Error & \multicolumn{3}{|c|}{ Step } \\
\hline & $10^{-2}$ & $d_{162}$ & $d_{176}$ & $d_{141}$ \\
\hline & $10^{-3}$ & $d_{1593}$ & $d_{1710}$ & $d_{1439}$ \\
\hline & $10^{-4}$ & $d_{15652}$ & $d_{16630}$ & $d_{14421}$ \\
\hline & $10^{-5}$ & $d_{123278}$ & $d_{132268}$ & $d_{111932}$ \\
\hline
\end{tabular}
for two concrete operators.

Table 5.1 
Example 5.1. Let $M=\mathbb{H}^{3}$ and let $T_{1}, T_{2}: M \rightarrow M$ be the nonexpansive mappings respectively defined by

$$
T_{1}(x)=\left(-x_{1},-x_{2},-x_{3}, x_{4}\right)
$$

and

$$
T_{2}(x)=\left(-x_{1}, x_{2}, x_{3}, x_{4}\right),
$$

for any $x=\left(x_{1}, x_{2}, x_{3}, x_{4}\right) \in \mathbb{H}^{3}$. Then $\operatorname{Fix}\left(T_{1}\right)=\{(0,0,0,1)\}$ and

$$
\operatorname{Fix}\left(T_{2}\right)=\left\{\left(x_{1}, x_{2}, x_{3}, x_{4}\right) \in \mathbb{H}^{3}: x_{1}=0, x_{2}^{2}+x_{3}^{2}=x_{4}^{2}-1\right\} .
$$

\begin{tabular}{|c|c|c|c|c|}
\hline \multicolumn{2}{|l|}{$T_{2}$} & $x_{0}^{1}$ & $x_{0}^{2}$ & $x_{0}^{3}$ \\
\hline \multirow{16}{*}{ MANN } & $\overline{\text { Step }}$ & \multicolumn{3}{|c|}{ Error } \\
\hline & $d_{1}$ & 1.85774905192289 & 2.14524665670207 & 1.44840325489716 \\
\hline & $d_{2}$ & 0.99079949435887 & 1.14413155024110 & 0.77248173594515 \\
\hline & $d_{3}$ & 0.61924968397430 & 0.71508221890069 & 0.48280108496572 \\
\hline & $d_{4}$ & 0.42462835472523 & 0.49034209296047 & 0.33106360111935 \\
\hline & $d_{5}$ & 0.30962484198715 & 0.35754110945034 & 0.24140054248286 \\
\hline & $d_{6}$ & 0.23590464151402 & 0.27241227386693 & 0.18392422284409 \\
\hline & $d_{7}$ & 0.18577490519229 & 0.21452466567021 & 0.14484032548972 \\
\hline & $d_{8}$ & 0.15012113550892 & 0.17335326518805 & 0.11704268726442 \\
\hline & $d_{9}$ & 0.12384993679486 & 0.14301644378014 & 0.09656021699315 \\
\hline & $d_{10}$ & 0.10393001689079 & 0.12001379897634 & 0.08102955272153 \\
\hline & Error & & Step & \\
\hline & $10^{-2}$ & $d_{26}$ & $d_{28}$ & $d_{23}$ \\
\hline & $10^{-3}$ & $d_{85}$ & $d_{91}$ & $d_{75}$ \\
\hline & $10^{-4}$ & $d_{271}$ & $d_{291}$ & $d_{239}$ \\
\hline & $10^{-5}$ & $d_{862}$ & $d_{926}$ & $d_{761}$ \\
\hline \multirow{16}{*}{ HALPERN } & Step & \multicolumn{3}{|c|}{ Error } \\
\hline & $d_{1}$ & 0.99837317911716 & 1.16992727222576 & 1.31340494917273 \\
\hline & $d_{2}$ & 0.71833797458614 & 0.83426922900920 & 0.97908824565578 \\
\hline & $d_{3}$ & 0.59032246197087 & 0.67290452208375 & 0.81786161285239 \\
\hline & $d_{4}$ & 0.47974944268260 & 0.54618472938029 & 0.67524807759001 \\
\hline & $d_{5}$ & 0.42202799259708 & 0.47635447878610 & 0.59506375226788 \\
\hline & $d_{6}$ & 0.36239751805204 & 0.40927663184813 & 0.51596963770002 \\
\hline & $d_{7}$ & 0.32928308480446 & 0.37005540327231 & 0.46796492723635 \\
\hline & $d_{8}$ & 0.29184728056858 & 0.32831699473056 & 0.41767189977732 \\
\hline & $d_{9}$ & 0.27026706878293 & 0.30305136210601 & 0.38570372433189 \\
\hline & $d_{10}$ & 0.24453550260074 & 0.27450025725934 & 0.35090005098115 \\
\hline & Error & \multicolumn{3}{|c|}{ Step } \\
\hline & $10^{-2}$ & $d_{151}$ & $d_{168}$ & $d_{218}$ \\
\hline & $10^{-3}$ & $d_{1524}$ & $d_{1702}$ & $d_{2198}$ \\
\hline & $10^{-4}$ & $d_{15266}$ & $d_{17050}$ & $d_{22001}$ \\
\hline & $10^{-5}$ & $d_{152673}$ & $d_{170513}$ & $d_{220033}$ \\
\hline
\end{tabular}

Table 5.2

For both algorithms we are going to consider the sequence of parameters $\alpha_{n}=$ $\frac{1}{n+3}$ for each $n=0,1, \cdots$, and the point 
$u=(0.60379247919382,0.27218792496996,0.19881426776106,1.21580374135624)$

for Halpern's iteration. We consider three random initial points $x_{0}$ :

$$
\begin{aligned}
& x_{0}^{1}=(0.69445440978475,1.01382609280137,0.99360871330745,1.87012527625153) ; \\
& x_{0}^{2}=(0.82054041398189,1.78729906182707,0.11578260956854,2.20932797928782) ; \\
& x_{0}^{3}=(0.93181457846166,0.46599434167542,0.41864946772751,1.50356127641534) .
\end{aligned}
$$

The numerical results are listed in the following tables, where $d_{n}=d\left(x_{n}, x^{*}\right)$ denote the error of the $n$th step with the unique fixed point $x^{*}=(0,0,0,1)$ in the first table for $T_{1}$; while, in the second one for $T_{2}, d_{n}=d\left(x_{n+1}, x_{n}\right)$ denote the distance between two consecutive iterates $x_{n+1}$ and $x_{n}$. In both tables we first compute, for both algorithm, the errors in the steps 1-10 of the iteration and later we provide the step where the errors get the lower bounds $10^{-2}, 10^{-3}, 10^{-4}$ and $10^{-5}$, respectively.

From the numerical results, one can observe that Mann's algorithm is much quicker than Halpern's algorithm. Moreover, as it is predicted by the theoretical results, the measure of the errors for the steps 1-10 in the first table show that the sequence of iterates is Fejer monotone just in the case of Mann algorithm.

\section{REFERENCES}

1. M. Bridson and A. Haefliger, Metric spaces of non-positive curvature, SpringerVerlag, Berlin, Heidelberg, New York, 1999.

2. F. E. Browder, Convergence of approximants to fixed points of nonlinear maps in Banach spaces, Arch. Rational Mech. Anal., 24 (1967), 82-90.

3. C. E. Chidume and C. O. Chidume, Iterative approximation of fixed points of nonexpansive mappings, J. Math. Anal. Appl., 318 (2006), 288-295.

4. M. P. DoCarmo, Riemannian Geometry. Boston: Birkhauser, (1992).

5. J. X. Da Cruz Neto, O. P. Ferreira and L. R. Lucambio Pérez, Contributions to the study of monotone vector fields, Acta Mathematica Hungarica, 94(4) (2002), 307-320.

6. O. P. Ferreira, L. R. Lucambio Pérez and S. Z. Nemeth, Singularities of monotone vector fields and an extragradient-type algorithm, Journal of Global Optimization, 31 (2005), 133-151.

7. O. P. Ferreira and P. R. Oliveira, Proximal point algorithm on riemannian manifolds, Journal of Optimization Theory and Applications, Optimization, 51(2) (2002), 257270.

8. J. Garcia-Falset, W. Kaczor, T. Kuczumov and S. Reich, Weak convergence theorems for asymptotically nonexpansive mappings and semigroups, Nonlinear Analysis, $\mathbf{4 3}$ (2001), 377-401. 
9. A. Genel and J. Lindenstrauss, An example concerning fixed points, Israel Journal of Mathematics, 22(1) (1975), 81-86.

10. K. Goebel and W. A. Kirk, Iteration processes for nonexpansive mappings, Contemporary Mathematics, 21 (1983), 115-123.

11. K. Goebel and S. Reich, Uniform Convexity, Hyperbolic Geometry, and Nonexpansive Mappings, Marcel Dekker, Inc., New York, 1984.

12. B. Halpern, Fixed Points of Nonexpanding Maps, Bull. Amer. Math. Soc., 73 (1967), 591-597.

13. S. Ishikawa, Fixed points and iteration of a nonexpansive mapping in a Banach space, Proc. Amer. Math. Soc., 59 (1976), 65-71.

14. T. H. Kim and H. K. Xu, Strong convergence of modified Mann iterations, Nonlinear Anal., 61 (2002), 51-60.

15. W. A. Kirk, Krasnoselskii's Iteration process in hyperbolic space, Numerical Functional Analysis and Optimization, 4(4) (1981/82), 371-381.

16. W. A. Kirk, Geodesic Geometry and Fixed Point Theory, Seminar of Mathematical Analysis (Malaga/Seville, 2002/2003), 195-225, Univ. Sevilla Secr. Publ., Seville, 2003.

17. M. A. Krasnosel'skij, Two remarks on the method of successive approximations (Russian), Uspehi Mat. Nauk., 10(63) (1955), 123-127.

18. P. Lions, Approximation des points fixes de contractions, C.R. Acad. Sci. Ser. A-B Paris, 284 (1977), 1357-1359.

19. W. R. Mann, Mean value methods in iteration, Proceedings of American Mathematical Society, 4(3) (1953), 506-510.

20. K. Nakajo and W. Takahashi, Strong convergence theorems for nonexpansive mappings and nonexpansive semigroups, J. Math. Anal. Appl., 279 (2003), 372-379.

21. S. Z. Nemeth, Variational inequalities on Hadamard manifolds, Nonlinear Analysis, 52 (2003), 1491-1498.

22. J. G. O'hara, P. Pillay and H. K. Xu, Iterative approaches to convex feasibility problems in Banach Space, Nonlinear Analysis, 64 (2006), 2002-2042.

23. S. Reich, Weak convergence theorems for nonexpansive mappings in Banach spaces, Journal of Mathematical Analysis and Applications, 67 (1979), 274-276.

24. S. Reich, Strong convergence theorems for resolvents of accretive operators in Banach spaces, Journal of Mathematical Analysis and Applications, 75 (1980), 287-292.

25. S. Reich and I. Shafrir, Nonexpansive iterations in hyperbolic spaces, Nonlinear Analysis, 15(6) (1990), 537-558.

26. T. Sakai, Riemannian Geometry, Translations of Mathematical Monographs 149, American Mathematical Society, Providence, RI, 1996. 
27. T. Suzuki, A sufficient and necessary condition for Halpern-type strong convergence to fixed point of nonexpansive mappings, Proceedings of American Mathematical Society, 135(1) (2007), 99-106.

28. C. Udriste, Convex Functions and optimization Methods on Riemannian Manifolds, Mathematics and its Applications 297, Kluwer Academics publishers, 1994.

29. R. Walter, On the metric projection onto convex sets in Riemannian spaces, Archiv der Mathematik, 25 (1974), 91-98.

30. R. Wittmann, Approximation of fixed points of nonexpansive mappings, Archiv der Mathematik, 58 (1992), 486-491.

31. H. K. Xu, Iterative algorithms for nonlinear operators, Journal of London Mathematical Society, 66 (2002), 240-256.

32. H. K. Xu, Fixed point algorithms: Recent progresses and applications, Plenary lecture in the 8th International Conference on Fixed Point Theory and Applications, 2007.

\section{Chong Li}

Department of Mathematics,

Zhejiang University,

Hangzhou 310027,

P. R. China

and

Department of Mathematics,

College of Sciences,

King Saud University,

P. O. Box 2455, Riyadh 11451,

Saudi Arabia

E-mail: cli@zju.edu.cn

Genaro López and Victoria Martín-Márquez

Departamento de Anlisis Matemático,

Universidad de Sevilla,

Apdo. 1160, 41080-Sevilla,

Spain

E-mail: glopez@us.es victoriam@us.es 